\title{
Controlled Helicity of the Dumbbell-shaped Supramolecular Assembly Triggered by Temperature
}

\author{
Shen Chen $^{1,2, *}$ \\ ${ }^{1}$ Department of Chemistry, Harbin Institute of Technology, Harbin 150001, China; \\ ${ }^{2}$ School of Science, Harbin University, Harbin 150086, China. \\ 63286277@qq.com
}

Keywords: dumbbell-shaped molecules, supramolecular chirality, self-assembly.

\begin{abstract}
We have demonstrated that self-assembled nanofibers can convert into a chiral state influnenced by external stimuli. This helicity convertion of the nonchiral assembly realizes in a reversible way on heating. Notably, this reversible nonchiral-chiral switching is accompanied by a transition between extended and contracted states in the elongated nanofibers.
\end{abstract}

\section{Introduction}

Recently, significant attention of supramolecular assembly has been paid to developing helical architectures with controlled chirality that mimic the structures ${ }^{[1-4]}$ and functions of biological helices. Among such studies, transfer of chiral information through the molecular assembly to construct elongated helical aggregates $\left[{ }^{5,6]}\right.$ is one of the highlighted topics. The majority of these studies focus on chiral transfer from chiral side chains to aromatic cores through molecular assembly. ${ }^{7-10]}$ There are, however, a few reports on the switching helical behavior influenced by external stimuli. Therefore, it is highly important to develop such stimulus-responsive materials and understand their behaviors.

\section{Experimental section.}

We present here the formation of aqueous nanofibers with switchable supramolecular chirality from self-assembly of dumbbell-shaped molecules (Figure 1a). The helicity of the nonchiral nanofibers is induced in a reversible way on heating. Furthermore, the chiral induction is accompanied by a transition from extended to contracted states of the elongated fibrillar aggregates. The detailed synthesis of the self-assembling dumbbell-shaped molecules, consisting of a rigid stem which is a carbazole end-capped hexa-p-phenylene and flexible chains which are chiral oligoether segments laterally attached to the carbazole units, is described in the supporting according to previously reported similar methods (Figure 1b). ${ }^{[11]}$

\section{Result and discussion}

Molecule 1, when dissolved in a suitable solvent for the oligother dendron, can self-assemble for its structural characteristics. And this behavior of $\mathbf{1}$ in aqueous solution was subsequently studied by absorption, emission spectroscopes, dynamic light scattering (DLS) and transmission electron microscopy (TEM). In chloroform solution $\left(1.8 \times 10^{-5} \mathrm{M}\right)$ the UV/Vis absorption spectrum of $\mathbf{1}$ shows a absorption maximum at $325 \mathrm{~nm}$ which is from the rod block (Figure S1a). In contrast, the maximum of absorption and the emission maximum in aqueous solution $\left(1.8 \times 10^{-5} \mathrm{M}\right)$ of $\mathbf{1}$ are both red-shifted, and the intensity is reduced, indicative of its aggregation behavior. The hydrodynamic radius $(\mathrm{RH})$ of the aggregates of $\mathbf{1}$ in aqueous solution $\left(1.8 \times 10^{-5} \mathrm{M}\right)$ was studied by using dynamic light scattering. It ranges from several nanometers to hundreds of nanometers (Figure 3a). The evidence for the formation of the aggregates was also provided by transmission electron microscopy (TEM). The TEM image of $\mathbf{1}$ with a negatively stained sample shows that the aggregates are 
elongated fibers with a uniform diameter of about $4.7 \mathrm{~nm}$ compared with the extended moleculer length (5.3 $\mathrm{nm}$ by CPK modeling) (Figure 2a), indicating the slice of each elementary cylindrical object with several micrometers lengths contains one molecule (Figure1b). These results suggest that molecule $\mathbf{1}$ in aqueous solution self-assembles into fibrillar aggregates.
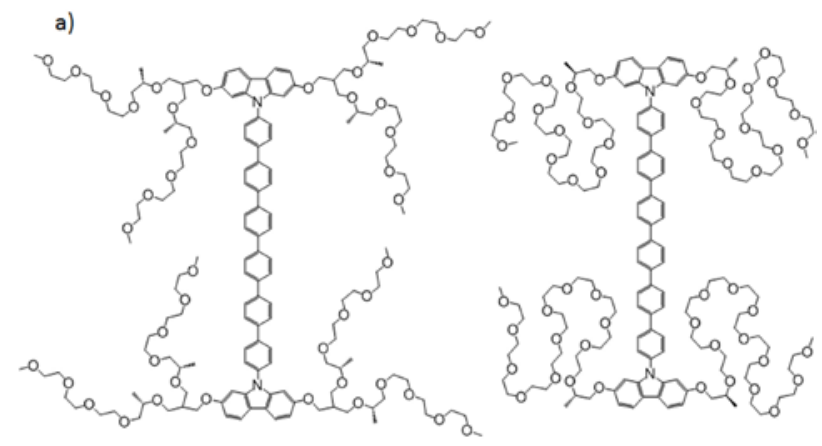

b)

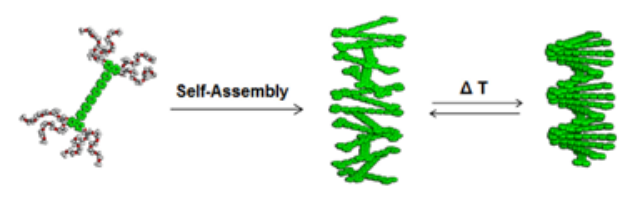

Fig.1 a) Molecular structure of dumbbell-shaped molecules 1 and 2. b)Schematic representation of the reversible transformation from achiral to chiral aggregates.
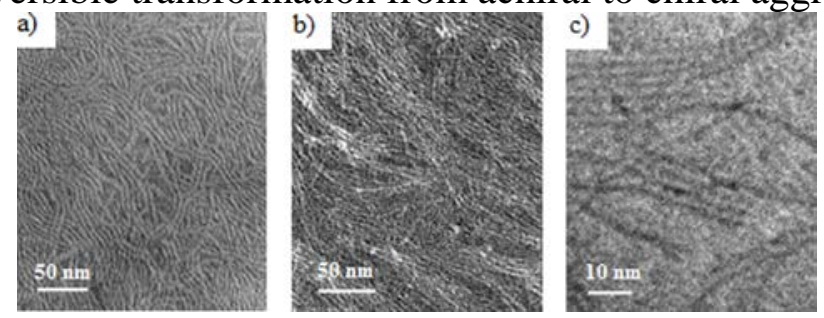

Fig. 2 TEM images of a) at $30{ }^{\circ} \mathrm{C}$, b) at $40{ }^{\circ} \mathrm{C}$ c) A cryo-TEM at $40{ }^{\circ} \mathrm{C}$ of aqueous solutions of $\mathbf{1}$.
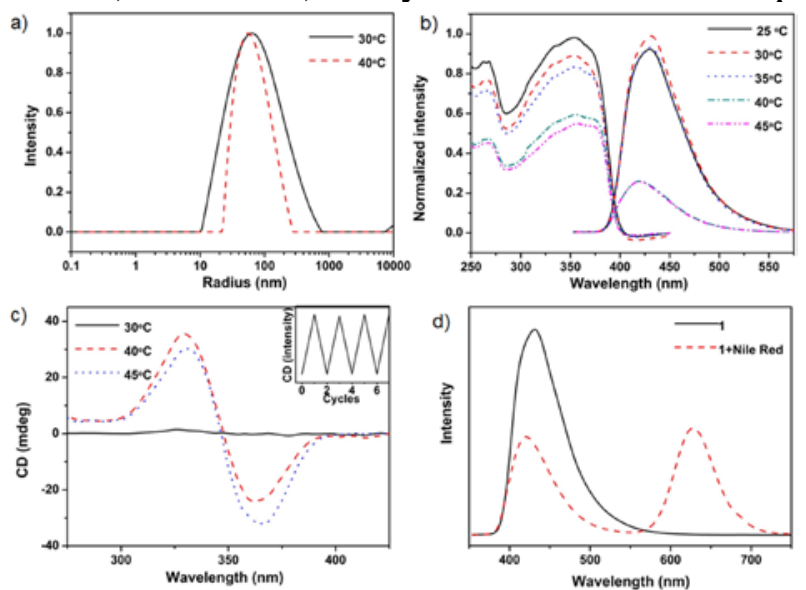

Fig. 3 a) DLS of $\mathbf{1}(0.01 \mathrm{wt} \%)$ at different temperatures. b) UV/Vis and fluorescence spectra of $\mathbf{1}(\mathrm{c}=$ $1.8 \times 10^{-5} \mathrm{M}$ ) with temperature variation. c) CD spectra at $330 \mathrm{~nm}$ in aqueous solution of $\mathbf{1}(\mathrm{c}=1.8 \times$ $10^{-5} \mathrm{M}$ ) (the inset is a CD intensity cycle). d) Fluorescence spectra of $\mathbf{1}\left(\mathrm{c}=1.8 \times 10^{-5} \mathrm{M}\right)$ with the addition of Nile Red (increase in molar ratio from 0 to 1 ) at $30{ }^{\circ} \mathrm{C}$ (excited at $350 \mathrm{~nm}$ ).

Notably, the CD spectra in aqueous solution of $\mathbf{1}$ at room temperature showed no signals, indicating that the fibrillar aggregates are nonchiral although 1 contains chiral side groups (Figure 3c).

For the aggregates are from the molecule 1 with hydrophilic oligoether flexible chains which may influenced by temperature because of their lower critical solution temperature (LCST) behavior, the influence of temperature on the aqueous solution was investigated. ${ }^{[12-14]} \mathrm{UV} / \mathrm{V}$ is transmittance (Figure S2a) shows the transition temperature was $36{ }^{\circ} \mathrm{C}$.As shown in TEM, the molecule 1 in aqueoussolution at higher temperatures above the LCST can also assemble into fibrillar objects with 
a shorten diameter of $4.5 \mathrm{~nm}$ (Figure 2b). The result was further confirmed by cryo-TEM and DLS measurements at $40{ }^{\circ} \mathrm{C}$ (Figure 2c and 3a). However, the CD spectra in aqueous solution of 1 above the transition temperature showed strong bisignated Cotton effect accompanied by notable changes in absorption and emission spectra (Figure $3 b$ and 3c), indicating the formation of the helix. ${ }^{15}$ The introduction of the chirality arises from the closer helical packing between the neighbored rods to reduce the steric constraints of aromatic segments because the flexible chains would be dehydrated upon heating.

Further evidence for the stacking interactions with temperature variation was provided by the fluorescence resonance energy transfer (FRET) experiments with Nile Red. The fluorescence spectra of 1 in aqueous solution containing Nile Red at $40^{\circ}$ Cshows a strong emission maximum at $430 \mathrm{~nm}$ corresponding to aggregated conjugated rods of $\mathbf{1}$ (Figure S2b). However, on cooling to room temperature which is below the LCST of molecule $\mathbf{1}$, the maximum intensity sharply decreases with the concomitant formation of an emission at $620 \mathrm{~nm}$, demonstrating that energy transfer occurs, indicative of larger distance between the adjacent rods within the fibers (Figure 3d). ${ }^{16}$ These results suggest that the fibril aggregates are lengthened because of the larger distance between the aromatic segments on cooling and the reason why the aggregates with chiral side chain are nonchiral is that the rod segments may rotate in a random way within the cores to block the transfer of chirality.

To corroborate the role of the oligoether chains in the helicity induction, 2 based on an easily stretchable linear oligo(ethylene oxide) chain with a similar volume fraction $\left(f_{\text {chain }}=0.78\right)$ was investigated. Similar to $\mathbf{1}$, the aggregates of molecule $\mathbf{2}$ are also fibres, as shown in TEM image (Figure S4). In great contrast to 1, 2 shows a significant Cotton effect even at room temperature that remains nearly unchanged above the LCST transition (Figure S3). These results strongly support that the branched oligoether chain with larger cross-section shows an important influnce in the stimuli-responsive nonchiral-chiral switch.

\section{Summary}

To summarize, we have demonstrated herein that the amphiphiles self-assemble into stimulus-responsive fibbers. The switchable chirality of the aggregates takes place in a reversible way through molecular reassemble on heating. Notably, this reversible nonchiral-chiral switching is accompanied by a transition between loosely-packed extended and close-packed contracted states in the elongated nanofibers. It is worth noting that these nanofibers were shown to recognize temperature by inducing supramolecular chirality.

\section{References}

[1]. Pijper D., Feringa B. L. Control of dynamic helicity at the macro- and supramolecular level. Soft Matter. Vol. 4(2008)No. 7, p. 1349-1372.

[2]. Hembury G. A., Borovkov V. V., Inoue Y. Chirality-sensing supramolecular systems. Chem. Rev. Vol.108( 2008), p.1-73.

[3]. Zhang J., Albelda M. T., Liu Y., Canary J. W. Chiral nanotechnology. Chirality. Vol. 17(2005)No. 7, p. 404-418.

[4]. Vera F., Serrano J. L., Sierra T. Twists in mesomorphic columnar supramolecular assemblies. Chem. Soc. Rev. Vol. 38(2009), p. 781-796.

[5]. Ajayaghosh A., Praveen V. K. . $\pi$-Organogels of Self-assembled p-phenylenevinylenes: soft materials with distinct size, Shape, and Functions. Acc. Chem. Res. Vol. 40(2007)No.8, p.644-656.

[6]. Brunsveld L., Zhang H., Glasbeek M., Vekemans J. A. J. M., Meijer E. W. Hierarchical growth of chiral self-assembled structures in protic media. J.. Am. Chem. Soc. Vol. 122(2000)No. 26, p. 6175-6182. 
[7]. Henze O., Feast W. J., Gardebien F., Jonkheijm P., Lazzaroni R., Philippe Leclère P., Meijer E. W., Schenning A. P. H. J. Chiral amphiphilic self-assembled $\alpha, \alpha^{6}$-linked quinque-, sexi-, and septithiophenes: synthesis, stability and odd-even effects. J. Am. Chem. Soc. Vol. 128( 2006)No.17, p. 5923-5929.

[8]. Chen S., Ma C., Huang Z., Lee M. Controlled helicity of the rigid-flexible molecular assembly triggered by water addition: from nanocrystal to liquid crystal gel and aqueous nanofibers. J. Phys. Chem. C. Vol. 118(2014)No. 15,p. 8181-8186.

[9]. Bae J., Choi J.-H., Yoo Y.-S., Oh N.-K., Kim B.-S., Lee M. Helical nanofibers from aqueous self-assembly of an oligo(p-phenylene)-based molecular dumbbell. J. Am. Chem. Soc. Vol. 127( 2005)No. 27, p. 9668-9669.

[10]. Ryu J.-H., Kim H.-J., Huang Z., Lee E., Lee M. Self-assembling molecular dumbbells: from nanohelices to nanocapsules triggered by guest intercalation. Angew. Chem., Vol. 118(2006)No. 32, p. 5430-5433.

[11]. Huang Z., Ryu J.-H., Lee E., Lee M. Tunable columnar organization by twisted stacking of end-capped aromatic rods. Chem. Mater. Vol. 19(2007)No. 26, p. 6569-6574.

[12]. Huang Z., Lee E., Kim H. J., Lee M. Aqueous nanofibers with switchable chirality formed of self-assembled dumbbell-shaped rod amphiphiles. Chem. Commun. (2009) ,p. 6819-6821.

[13]. Dormidontova E. E. Role of competitive PEO-water and water-water hydrogen bonding in aqueous solution PEO behavior. Macromolecules. Vol. 35(2002)No. 3, p. 987-1001.

[14]. Smith G. D., Bedrov D. Roles of enthalpy, entropy, and hydrogen bonding in the lower critical solution temperature behavior of poly(ethylene oxide)/water solutions. J. Phys. Chem. B. Vol. 107(2003)No. 14, p. 3095-3097.

[15]. Jonkheijm P., Hoeben F. J. M., Kleppinger R., Van Herrikhuyzen J., Schenning A. P. H. J., Meijer E. W. Transfer of $\pi$-conjugated columnar stacks from solution to surfaces. J. Am. Chem. Soc., 2003, 125(51), p. 15941-15949.

[16]. Lee E., Kim J.-K., Lee M. Lateral association of cylindrical nanofibers into flat ribbons triggered by “molecular glue”. Angew. Chem. Int. Ed. Vol. 47( 2008)No. 34, p. 6375-6378. 